\title{
Rosa canina as an adjunctive treatment of asthma: A hypothesis
}

Samin Hamidi ${ }^{1,2}$, Haleh Vaez ${ }^{1,3}$, Parina Asgharian ${ }^{1,4^{*}}$

1. Student research committee, Tabriz University of Medical Sciences

2. Department of Medicinal Chemistry, Faculty of Pharmacy, Tabriz University of Medical Sciences

3. Department of Pharmacology, Faculty of Pharmacy, Tabriz University of Medical Sciences

4. Department of Pharmacognosy, Faculty of Pharmacy, Tabriz University of Medical Sciences

\begin{abstract}
Asthma is one of the most common chronic disorders and about $7 \%$ of the US population suffers from asthma. Initial response to allergy is associated with mediators like histamine, prostaglandin (PG) $D_{2}$ and leukotriene (LT) $\mathrm{C}_{4}$. In asthmatic patients, 5-lipo-oxygenase (5-LO) pathway produces more $\mathrm{LTC}_{4}$ than the healthy people. Also, pro-inflammatory factors like interleukin (IL)-1, IL-6 and Tumor Necrosis Factor (TNF)- $\alpha$ play a remarkable role in bronchoconstriction. The common asthma medications are associated with a number of unwanted side effects, especially throughout long term treatment. Herbal medication is an alternative treatment for asthma and the plants are rich sources of antioxidants that intensively reduce the activity of free radicals. Among several herbs, Rosa canina $(R C)$ is a potent anti-inflammatory remedy which contains a number of well-known antioxidants including vitamin C, quercetin, hesperidin, rutin and some other therapeutic compounds. The aim of this study was to review the scientific evidences supporting the probable influence of Rosa canina on asthma.
\end{abstract}

Keywords: Asthma; Rosa canina; Anti-inflammatory

\section{Introduction}

Rosa canina (RC) L. (rosehip) is a species from the Rosaceae family, commonly known as the dog rose. It is a widespread shrub in Europe, northwest parts of Africa and western Asia. Traditionally, the medicinal benefits of $\mathrm{RC}$ have been known for decades. RC is a rich source of vitamin $C$ and it was used as syrup during the World War II because of its preventive effect on the scurvy (1).

This plant is introduced in folk medicine as diuretic, laxative, anti-arthritis, anti-gout, antifever and cold and also as a cure for vitamin C deficiency (2).

\section{Corresponding author:}

Parina Asgharian

Faculty of Pharmacy, Tabriz University of Medical Sciences Daneshgah St. Tabriz-Iran P.O.Box: 51664-14766 Email: Parina.asgharian@gmail.com

Receive date: 2014-08-17| Accept date: 2014-09-01| Publish date: 2014-09-21

DOI: 10.7575/aiac.abcmed.15.03.01.14

\section{Al}


Investigations

on

phytochemical constituents of RC have been reported several valuable active ingredients such as phenolic acids, proanthocyanidins, tannins, flavonoids [flavones, flavonol, flavanone], dihydrochalcone derivatives, unsaturated and polyunsaturated fatty acids, phospholipids, minerals, galactolipids and carotenoids $(2,3)$.

It was evaluated that active compounds of RC have anti-inflammatory and anti-oxidant properties in vitro 4 and in vivo (5). Also, the inhibitory effect of a number of active compounds such as flavonoids, isoflavones and chalcones on the cyclooxygenase (COX) and/or 5-lipooxygenase (5-LO) pathways have been demonstrated (6).

Tannins and other compounds in rose-hip might contribute to its anti-inflammatory potentials. Antioxidant power of RC has been indicated in the literature (7). Especially, galactolipid isolated from rosehip has been confirmed to inhibit chemotaxis of peripheral blood neutrophils (4). There is a significant and direct association of vitamin C, vitamin E, and lutein/zeaxanthin intake with proper pulmonary function (8). Despite the effectiveness of RC on respiratory diseases due to its active compounds, this efficacy has not yet been studied. The authors would like to propose a hypothetic correlation between antiasthma potential of $\mathrm{RC}$ and related antiinflammatory and antioxidant constituents.

\section{Asthma}

Asthma is a disorder that is caused by chronic inflammation in the airway. Asthma attack symptoms include: cough, wheezing, breathing difficulties and chest tightness (9). The number of people in the U.S. with asthma is growing. One in 12 people had asthma in 2009, compared with 1 in 14 in 2001, according to the Center for Disease Control and Prevention (CDC) report. Descriptions of the mechanisms of asthma have been in circulation for decades. Improved understanding of the asthma mechanism is fundamental for optimal management of asthma and may lead to novel approaches. Airway inflammation is a key mechanism in airway disorders. 5- LO is expressed and produces more leukotriene (LT) C4 than normal condition. There are other inflammatory mediators that are involved in asthma such as cytokines, Ig $\mathrm{E}$ and histamine (10). Allergic asthma is characterized by airway hyper responsiveness and inflammation of the airway with remarkable over-expression of inducible nitric oxide synthase (iNOS) and overintroduction of nitric oxide (NO). INOS gene is associated with severity of asthma (11).

Bronchial asthma usually is accompanied with increase in vascular permeability due to the over-expression of receptor activator of nuclear factor-kappa B (NF-kB) (12). The modifying influence of antioxidant on NF-KB (RANK) expression and modulation of vascular permeability have been proven (13)

One of the most major symptoms of asthma is chronic cough. The mechanism by which cough occurs is not fully understood. Various categories of antitussive medicines like opioids, anti-histamines and expectorants wildly have been used. Some of these drugs have side effects like dependency for opioids and anticholinergic effect for antihistamines. Therefore, due to the above-mentioned side effects, many attempts have been evaluate to search therapeutic effect of natural plant component with decreased side effects.

\section{Hypothesis}

Although there are a large number of researches indicating a progress in asthma treatment, local and systematic adverse effects of inhaled corticosteroids lead to alternative and complementary remedies. Herbal remedies, because of their effective constituents, have been accepted as the third 
popular treatment in asthma (14). The investigations about the effect of herbal remedy in asthma treatment are still on improvement and there is high evidence on the usage of herbal medicines in asthma. Chinese anti-asthma herbal medicine appears to be a safe alternative medicine (15).

Presence of some fundamental ingredients in $\mathrm{RC}$ is responsible for its traditional usage for alleviating some diseases such nephrolithiasis, inflammatory disorders, cold and cough. Carotenoid compositions of RC have been investigated; major carotenoids are betacarotene, lycopene, beta-chryptoxanthin, rubixanthin, zeaxanthin and lutein (16). Lycopene (also found in RC) is one of the most important antioxidant agents (17). It is believed that oxidative stress, especially lipid peroxidation, is involved in pathophysiology of asthma. Additionally, it is investigated that inflammatory cells like eosinophils can produce Reactive Oxygen Species (ROS) when trigged by allergens or air pollution (18). ROS, released from activated cells or during phagocytosis that are present in bronchoalveolar lavage, can serve as chemical mediators of anaphylaxis. Oxygen-free radicals play their role through different ways like increasing epithelial shedding, contraction of smooth muscle cells, functional impairment of beta-adrenoceptors, pulmonary vasoconstriction and the vascular permeability in the airway structures (19). It has been reported that oxidative stress in asthma can diminish with anti-oxidants (20). Results present that lycopene-rich supplements show a protective effect against exerciseinduced asthma (21).

Another study indicates that lycopene modifies non-eosinophilic airway inflammation in asthma (22). Evidences confirm that lycopene can protect cells from inflammation via its redox-based property on suppression of NF-KB, which is a key factor that facilitates the production of inflammatory biomarkers. Hence, the authors suggest the probable effect of lycopene on reducing asthma inflammation.

Antioxidant and anti-inflammatory properties of proanthocyanidins, a group of polyphenolics called flavanols, have been known for years (23). Zhou et al. demonstrated that the grape seed proanthocyanidine (GSPE) extract not only intensively decreases the expression of iNos in the lung tissues, IL4 and IL13, but also increases the levels of IFN (Interferon) $\gamma$ in BALB/C mice (24). The role of GSH redox cycle in asthma development has also been studied (25). In addition, researchers demonstrated that GSPE from grape seed significantly suppresses formation of proinflammatory cytokines and lipid peroxidation26. It seems that antiinflammatory effects of RC might be used in asthma in whose pathogenesis inflammation plays a major role. Bioflavonoids reduce serum leukotrienes and are therefore recommend in chronic asthma treatment (27). Phenolic acids, tannic acid and quercetin have an inhibitory role in lipid peroxidation in asthma inflammation (28). Some botanical extracts like RC, Salix alba and Nettle leaf are of antiinflammatory effects such as inhibiting IL-1 $\beta$ induced NF-KB activation (29).

The galactolipid (2S)-1,2-di-O-[(9Z,12Z,15Z)octadeca-9,12,15-trienoyl]-3-O-beta-d galactopyranosyl glycerol isolated from $\mathrm{RC}$ is an anti-inflammatory agent with inhibitory effects on chemotaxis of human peripheral blood neutrophils (30). Through these mechanisms the anti-asthma effects of RC might be proposed.

The effectiveness of plant phytochemicals has been studied. It is accepted that there is a direct correlation between bronchodilating potency and cough inhibition. 31 It has been elucidated that prenylated flovonoids have a significant role on cyclooxygenase (COX)-1, (COX)-2, 5-lipoxygenase (5-LOX) and 12-LOX due to their anti-inflammatory effects. It has 
also been revealed that flavonoids can inhibit cyclooxygenase and lipooxygenase in arachidonic methabolism cycle. 320ther aspects of anti-asthmatic activity of flavonoids have been emphasized in another study. Quercetin is a common flavonoid which prevents the Tumor Necrosis Factor (TNF)- $\alpha$ generation and its gene expression in a dose dependent manner (33).
Above-mentioned evidences suggest that there is a close correlation between asthma, inflammation and oxidative stress. Further studies are required to elucidate the efficacy of this plant in patients who suffer from respiratory disease and also the mechanisms through which this herb can help millions of people affected by asthma.

\section{References}

1. Haas LF. Rosa canina (dog rose). J Neurol Neurosurg Psychiatry 1995;59(5):470.

2. Chrubasik C, Roufogalis BD, Muller-Ladner U, Chrubasik S. A systematic review on the Rosa canina effect and efficacy profiles. Phytother Res 2008;22(6):725-733.

3. Altiner $\mathrm{D}$, Kilicgun $\mathrm{H}$. Correlation between antioxidant effect mechanisms and polyphenol content of Rosa canina. Phcog Mag 2010;6:238-241.

4. Kharazmi A, Winther K. Rose hip inhibits chemotaxis and chemiluminescence of human peripheral blood neutrophils in vitro and reduces certain inflammatory parameters in vivo. Inflammopharmacology 1999;7(4):377-386.

5. Deliorman Orhan D, Hartevioğlu A, Küpeli E, Yesilada E. In vivo anti-inflammatory and antinociceptive activity of the crude extract and fractions from Rosa canina L. fruits. J. Ethnopharmacol 2007;112(2):394-400.

6. Kupeli E, Orhan I, Toker G, Yesilada E. Anti-inflammatory and antinociceptive potential of Maclura pomifera (Rafin.) Schneider fruit extracts and its major isoflavonoids, scandenone and auriculasin. J. Ethnopharmacol 2006;107(2):169-174.

7. Wenziga E.M, Widowitza U, Kunertb O, Chrubasikc S, Bucara F, Knaudera E, Bauer R. Phytochemical composition and in vitro pharmacological activity of two rose hip (Rosa canina L.) preparations. Phytomedicine 2008;15(10):826-835.

8. Schunemann H.J, McCann S, Grant B.J.B, Trevisan M, Muti P, Freudenheim J.L. Lung function in relation to intake of carotenoids and other antioxidant vitamins in a population-based study. Am J Epidemiol 2002;155(5):463-471.

9. Meren M, Pruljan L.J, Loit H.M, Polluste J, Jonsson E, Kiviloog J, Lundback B. Asthma, chronic bronchitis and respiratory symptoms among adults in Estonia according to a postal questionnaire. Respiratory Medicine 2001;95(12):954-964.

10. Chung KF, Barnes PJ. Cytokines in asthma. Thorax 1999;54(9):825-857.

11. Batra J, Singh T.P, Mabalirajan U, Sinha A, Prasad R, Ghosh B. Association of inducible nitric oxide synthase with asthma severity, total serum immunoglobulin $E$ and blood eosinophil levels. Thorax 2007;62(1):16-22.

12. Min J.K, Kim Y.M, Kim Y.M, Kim E.C, Gho Y.S, Kang I.J, Lee S.Y, Kong Y.Y, Kwon Y.GI. Vascular endothelial growth factor up-regulates expression of receptor activator of NF-kappa B (RANK) in endothelial cells. Concomitant increase of angiogenic responses to RANK ligand. J Biol Chem 2003;278(41):39548-39557.

13. Lee KS. An antioxidant modulates expression of receptor activator of NF-kappaB in asthma. Exp Mol Med. 2006;38(3):217-229.

14. Singh B.B, Khorsan R, Vinjamury S.P, Der-Martirosian C, Kizhakkeveettil A, Anderson T. Herbal treatments of asthma: a systematic review. J Asthma 2007;44(9):685-698.

15. Srivastava K.D, Kattan J.D, Zou Z.M, Li J.H, Zhang L, Wallenstein S, Goldfarb J, Sampson H-A, Li X.M. The Chinese herbal medicine formula FAHF-2 completely blocks anaphylactic reactions in a murine model of peanut allergy. Journal of allergy and clinical immunology(JACI). 2005;115(1):171-178. 
16. Hodisan T, Socaciu C, Ropan I, Neamtu G. Carotenoid composition of Rosa canina fruits determined by thinlayer chromatography and high-performance liquid chromatography. J Pharm Biomed Anal 1997;16(3):521528.

17. Reifen R, Nur T, Matas Z, Halpern Z. Lycopene supplementation attenuates the inflammatory status of colitis in a rat model. Int J Vitam Nutr Res 2001;71(6):347-351.

18. Wood LG, Gibson PG, Garg ML. Biomarkers of lipid peroxidation, airway inflammation and asthma. Eur Respir J 2003;21(1):177-186.dard, P., Damon M, Cluzel M, Bousquet J, Chanez P, Crastes De Paulet A, Michel F-B.

19. Godard P, Damon M, Cluzel M, Bousquet J, Chanez P, Crastes De Paulet A, Michel F-B. Oxygen free radicals and bronchial asthma. Allerg Immunol (Paris) 1987;19(8):15-18.

20. Rahman I, Morrison D, Donaldson K, MacNee W. Systemic oxidative stress in asthma, COPD, and smokers. Am J Respir Crit Care Med 1996;154(4 Pt 1):1055-1060.

21. Neuman I, Nahum H, Ben-Amotz A. Reduction of exercise-induced asthma oxidative stress by lycopene, a natural antioxidant. Allergy 2000;55(12):1184-1189.

22. Wood LG, Garg ML, Powell H, Gibson PG. Lycopene-rich treatments modify noneosinophilic airway inflammation in asthma: proof of concept. Free Radic Res 2008;42(1):94-102.

23. Bagchi D, Garg A, Krohn R.L, Bagchi M, Tran M.X, Stohs S.J. Oxygen free radical scavenging abilities of vitamins $\mathrm{C}$ and $\mathrm{E}$, and a grape seed proanthocyanidin extract in vitro. Res Commun Mol Pathol Pharmacol 1997;95(2):179-189.

24.Zhou D.y, Du Q, Li R.R, Huang M, Zhang Q, Wei G.Z. Grape seed proanthocyanidin extract attenuates airway inflammation and hyperresponsiveness in a murine model of asthma by downregulating inducible nitric oxide synthase. Planta medica 2011;77(14):1575-1581.

25. Fitzpatrick AM, Jones DP, Brown LA. Glutathione redox control of asthma: from molecular mechanisms to therapeutic opportunities. Antioxid Redox Signal 2012;17(2):375-408.

26. Li WG, Zhang XY, Wu YJ, Tian X. Anti-inflammatory effect and mechanism of proanthocyanidins from grape seeds. Acta Pharmacol Sin 2001;22(12):1117-1120.

27. Hosseini S, Pishnamazi S, Sadrzadeh S.M.H, Faridi F, Farid R, Watson R.R Pycnogenol (R) in the Management of Asthma. J Med Food 2001;4(4):201-209.

28. Cholbi MR, Paya M, Alcaraz MJ. Inhibitory effects of phenolic compounds on CCl4-induced microsomal lipid peroxidation. Experientia 1991;47(2):195-199.

29. Shakibaei M, Allaway D, Nebrich S, Mobasheri A. Botanical Extracts from Rosehip (Rosa canina), Willow Bark (Salix alba), and Nettle Leaf (Urtica dioica) Suppress IL-1beta-Induced NF-kappaB Activation in Canine Articular Chondrocytes. Evid Based Complement Alternat Med 2012;2012:509383.

30. Larsen E, Kharazmi A, Christensen LP, Christensen SB. An antiinflammatory galactolipid from rose hip (Rosa canina) that inhibits chemotaxis of human peripheral blood neutrophils in vitro. J Nat Prod 2003;66(7):994995.

31. Chung KF. Drugs to suppress cough. Expert Opin Investig Drugs 2005;14(1):19-27.

32. Chi Y.S, Jonga H.G, Sonb K.H, changc H.W, Kangd S.S, Kima H.P. Effects of naturally occurring prenylated flavonoids on enzymes metabolizing arachidonic acid: cyclooxygenases and lipoxygenases. Biochem Pharmacol 2001;62(9):1185-1191.

33. Nair M.P, Mahajan S, Reynolds J.L, Aalinkeel R, Nair H, Schwartz S.A, Kandaswami C. The flavonoid quercetin inhibits proinflammatory cytokine (tumor necrosis factor alpha) gene expression in normal peripheral blood mononuclear cells via modulation of the NF-kappa beta system. Clin Vaccine Immunol 2006;13(3):319-32. 